Laser System for Photoelectron and $X$-Ray Production in the PLEIADES Compton Light Source

D. J. Gibson, C. P. J. Barty, S. M. Betts, J. K. Crane, I. Jovanovic

April 25, 2005

2005 Particle Accelerator Conference Knoxville, TN, United States

May 16, 2005 through May 20, 2005 
This document was prepared as an account of work sponsored by an agency of the United States Government. Neither the United States Government nor the University of California nor any of their employees, makes any warranty, express or implied, or assumes any legal liability or responsibility for the accuracy, completeness, or usefulness of any information, apparatus, product, or process disclosed, or represents that its use would not infringe privately owned rights. Reference herein to any specific commercial product, process, or service by trade name, trademark, manufacturer, or otherwise, does not necessarily constitute or imply its endorsement, recommendation, or favoring by the United States Government or the University of California. The views and opinions of authors expressed herein do not necessarily state or reflect those of the United States Government or the University of California, and shall not be used for advertising or product endorsement purposes. 


\title{
LASER SYSTEM FOR PHOTOELECTRON AND X-RAY PRODUCTION IN THE PLEIADES COMPTON LIGHT SOURCE*
}

\author{
D. J. Gibson", C. P. J. Barty, S. M. Betts, J. K. Crane, I. Jovanovic, LLNL, Livermore, CA 94551, \\ U.S.A.
}

Abstract

The PLEIADES (Picosecond Laser-Electron Interaction for the Dynamic Evaluation of Structures) facility provides tunable short $\mathrm{x}$-ray pulses with energies of $30-140 \mathrm{keV}$ and pulse durations of $0.3-5$ ps by scattering an intense, ultrashort laser pulse off a 35-75 $\mathrm{MeV}$ electron beam. Synchronization of the laser and electron beam is obtained by using a photoinjector gun, and using the same laser system to generate the electrons and the scattering laser. The Ti:Sapphire, chirped pulse amplification based $500 \mathrm{~mJ}, 50 \mathrm{fs}, 810 \mathrm{~nm}$ scattering laser and the similar $300 \mu \mathrm{J}, 5 \mathrm{ps}, 266 \mathrm{~nm}$ photoinjector laser systems are detailed. Additionally, an optical parametric chirped pulse amplification (OPCPA) system is studied as a replacement for part of the scattering laser front end. Such a change would significantly simplify the set-up the laser system by removing the need for active switching optics, as well as increase the pre-pulse contrast ratio which will be important when part of the scattering laser is used as a pump beam in pump-probe diffraction experiments using the ultrashort tunable $\mathrm{x}$-rays generated as the probe.

\section{INTRODUCTION}

The PLEIADES (Picosecond Laser-Electron InterAction for the Dynamic Evaluation of Structures) facility is designed to produce ultrashort, high brightness $\mathrm{x}$-ray pulses with tunable photon energies from 20-200 $\mathrm{keV}$ through the Thomson scattering of a high-intensity laser off a high-brightness electron beam. This requires the simultaneous integration of two laser systems and an $\mathrm{S}$-Band RF photoinjector-based linac at Lawrence Livermore National Laboratory (LLNL). A block diagram of the overall system is shown in Figure 1. Thus far, we have successfully demonstrated a flux of $\sim 10^{7}$ photons/shot, which corresponds roughly to a brightness of $10^{16}$ photons $/ \mathrm{mm}^{2} / \mathrm{s} / \mathrm{mrad}^{2} / 0.1 \% \mathrm{BW}$, at energies from 40-140 keV [1]. PLEIADES uses a titanium-doped sapphire (Ti:Sapphire)-based chirped-pulse-amplification (CPA) laser system to produce both $500 \mathrm{~mJ}$ of compressed 820-nm laser light to scatter off the electron beam and up to $1.2 \mathrm{~mJ}$ of $266 \mathrm{~nm}$ light to generate the electron beam in the photoinjector. Here, an overview of these two laser systems is presented as well as initial investigations into a hybrid chirped-pulse amplification scheme to replace the regenerative amplifier based front

* This work was performed under the auspices of the U.S. Department of Energy by University of California, Lawrence Livermore National Laboratory under Contract W-7405-Eng-48.

"gibson23@1ln1.gov end of the CPA system.

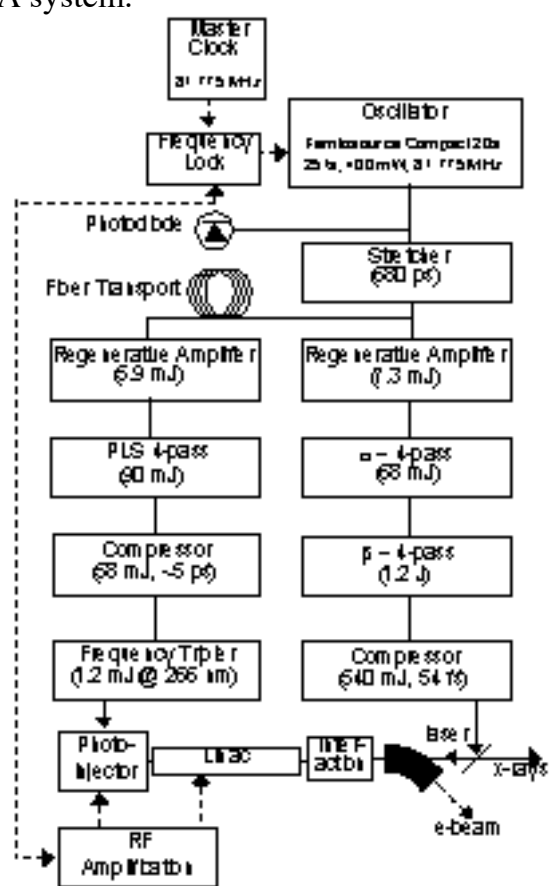

Figure 1:Block Diagram of the PLEIADES system

\section{LASER SYSTEMS}

The laser system begins with a mirror-dispersioncontrolled[2], Kerr-lens mode-locked, Compact C20s Ti:Sapphire oscillator from Femtosource, which produces $30 \mathrm{fs}, 5.5 \mathrm{~nJ}$ pulses with a bandwidth of $37 \mathrm{~nm}$ centered at $818 \mathrm{~nm}$ with a repetition rate of $81.557 \mathrm{MHz}$ (the $35^{\text {th }}$ subharmonic of the S-Band operational wavelength of the accelerator). This pulse train also serves as the master triggering signal for all the linac timing systems, minimizing jitter between the laser and rf systems. The pulse train is sent into an all-reflective parabolic mirror based expander[3], then split with a dielectric beam splitter into two beams with $30 \%$ of the light being coupled into a fiber to seed the photoinjector laser and the remaining $70 \%$ used to seed the high-intensity scattering laser. Because the same oscillator seeds both laser systems, minimal timing jitter between the systems is assured.

\section{Photoinjector Laser}

The phototoinjector laser is seeded through a 50-meter, single-mode fiber. After coupling and transport losses, the seed light has $90 \mathrm{pJ}$ per pulse, which is coupled into a linear regenerative amplifier (regen) cavity pumped with $50 \mathrm{~mJ}$ of 532-nm light. This produces $5.9 \mathrm{~mJ}$ IR pulses at 
$10 \mathrm{~Hz}$. Following the regen is a bow-tie configuration 4pass power amplifier pumped with $280 \mathrm{~mJ}$ of $532-\mathrm{nm}$ light which amplifies the cavity output to $90 \mathrm{~mJ}$ with measured $\mathrm{M}^{2}$ values of 1.15 and 1.36 in the two axes. This light is then sent into the pulse compressor.

Due to the disruptive effects of Coulomb repulsion from the electrons that results from high electron density, simulations show that the best quality beams result from using laser pulses with lengths of a few ps to generate the electrons. To create this few-picosecond pulse from the broad-bandwidth oscillator, the photoinjector laser is not fully compressed to the transform limit, leaving a slightly chirped pulse. This also allows us to bypass the problem raised by propagating the laser through 50 meters of fiber, which produces significant cubic phase errors in the pulse that cannot be corrected with a standard pulse compressor; because the pulse is not being fully compressed, the quadratic phase dominates this residual cubic phase from the fiber. The pulse length is left at $7 \mathrm{ps,}$ measured using a streak camera with 300 fs resolution[4] Following compression, the pulse is frequency-doubled and tripled in a pair of Type I BBO crystals. This UV pulse is clipped with an aperture to a diameter of $2 \mathrm{~mm}$ to provide a hard-edged UV spot, which improves the emittance of the generated electron beam. The aperture plane is relay imaged $\sim 50 \mathrm{~m}$ to the photoinjector cathode. Generally, about $1 \mathrm{~mJ}$ of UV light is available at the photocathode; to prevent damage to the cathode the system is often turned down to provide only about $270 \mu \mathrm{J}$.

\section{Scattering Laser}

Following the beam splitter that separates the light for the UV laser and the high-intensity scattering system, the pulse train is injected into a regen pumped by $45 \mathrm{~mJ}$ of $532-\mathrm{nm}$ light and amplified to $7 \mathrm{~mJ}$ at $10 \mathrm{~Hz}$. This is followed by two four-pass bow-tie power amplifiers. The first, pumped with $200 \mathrm{~mJ}$ of 532-nm light, gives $63 \mathrm{~mJ}$ of energy. The second, pumped with a total of $2.7 \mathrm{~J}$ of 532-nm light from two lasers, produces $1.2 \mathrm{~J}$ of uncompressed IR light with $M^{2}$ values of 1.64 and 1.58 along the two axes. The laser is relay imaged $46.5 \mathrm{~m}$ to the compressor, which compresses the beam to $55 \mathrm{fs}$. The compressed pulse then propagates $20 \mathrm{~m}$ to the final focusing optics. The transmission through the transport and compressor is $45 \%$, leaving up to $540 \mathrm{~mJ}$ available in the interaction region for Thomson scattering.

To perform dynamic experiments, a third laser is required, to pump the sample under test that would be probed with the $\mathrm{x}$-rays. To produce this beam, a small sample of the main scattering beam is extracted. The final turning mirror before the laser focus has two holes drilled in it, to allow both the core of the laser beam and the core of the x-ray beam to pass through it unimpeded. Running at full power, with $540 \mathrm{~mJ}$ of IR light reaching the interaction region, approximately $25 \mathrm{~mJ}$ of light will pass through the $5 \mathrm{~mm}$ hole in the mirror. The portion of the laser that passes through the mirror will then be upcollimated with a pair of lenses, and sent through a window out of the vacuum chamber. A waveplate and polarizer will be used to adjust the laser energy getting to the crystal. A delay stage will be used to adjust the timing of the laser arrival relative to the x-rays. Meanwhile, the $\mathrm{x}$-rays will propagate through the final turning mirror and leave the vacuum via a $300 \mu \mathrm{m}$ beryllium window. They will diffract off the sample under study and be detected by the x-ray CCD camera. This layout is shown in Figure 2 .

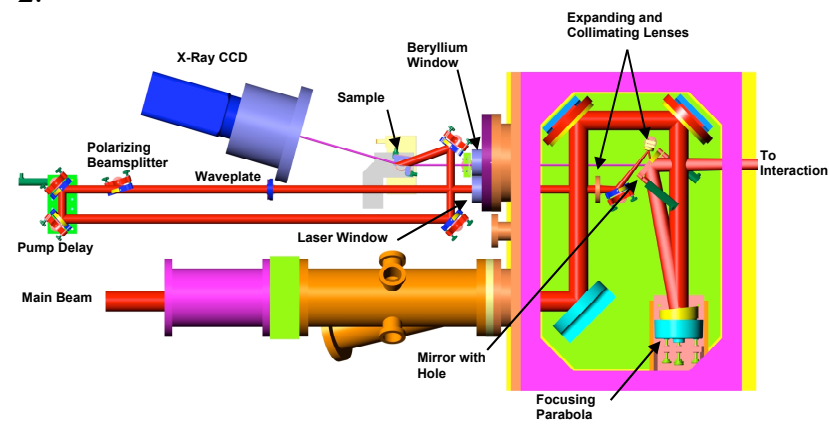

Figure 2: Experimental set-up for the initial dynamic diffraction experiment

\section{HYBRID CPA}

An alternative to traditional laser amplification, where a gain medium stores energy to transfer to a laser pulse passing through, is a process known as "optical parametric amplification", wherein a pump laser pulse is mixed in a nonlinear crystal with a seed pulse to be amplified. Through difference-frequency generation, the pump laser photons are converted to two lower-frequency photons, one matching the frequency of incoming seed beam (thereby amplifying it), and one at a corresponding frequency to maintain energy conservation. This has advantages over standard laser amplification in that it allows gain at a wide variety of wavelengths based on angle and/or temperature tuning of the non-linear crystal, as opposed to the electronic level structure of the gain medium which is fixed for a given material. Also, a parametric system can also have very wide gain bandwidths, which implies it can be used to amplify chirped pulses[5]. This process is referred to as Optical Parametric Chirped Pulse Amplification (OPCPA).

For the PLEIADES system, there are additional benefits from using OPCPA for amplification, which suggest it might be an excellent candidate for replacement of regens. First, the optical nonlinearities of some materials are high enough to allow gain on the order of $10^{4}-10^{5}$ in just a single pass of a few $\mathrm{cm}$ of material. This would eliminate the complicated multiple-pass schemes to get large amounts of gain that necessitate regenerative amplifiers. The need to construct a cavity and switch the laser light into and out of it would be eliminated, removing considerable complexity in the system. Second, in a cavity, each time the pulse passes the switch-out point a small $(\sim 1 \%)$ amount of light leaks out. This results in a train of pulses at the exit of the amplifier spaced several nanoseconds apart. As these pre-pulses propagate through the laser chain, they can be amplified by subsequent stages. For experiments where this beam will 
strike a target, these prepulses can have a significant impact by ablating or pre-ionizing the material before the arrival of the main pulse, significantly affecting the physics of the interaction. In an OPCPA system, however, the only pulse that sees any amplification is the pulse coincident with the pump beam; the rest of the pulse-train is not amplified. The prepulse contrast then matches the gain in the OPCPA stage. Since gain is on the order of $10^{5}$, this is already a thousandfold improvement over the regen contrast. Furthermore, a scheme known as cascaded optical parametric amplification (COPA) has been proposed[6] which could have essentially infinite prepulse contrast.

The downside of this process is that, since gain is only realized when the pump and seed pulses are coincident in both space and time, the pulse length mismatch between the seed beam of a CPA laser system (100s of ps) and a commercial Q-switched pump laser $(\sim 7 \mathrm{~ns})$ means most of the pump energy is wasted, giving efficiencies on the order of $1-2 \%$. However, once a commercial pump laser signal has been used in an OPCPA stage to amplify a signal beam, all the unused pump light (less surface reflections) is still available. This is in contrast to a laser amplifier where the pump light is absorbed by the gain medium and, if unused, is lost to heating of the medium. This leftover pump light can be used to pump a traditional laser amplifier, and a multipass amplifier can be used to further amplify the signal pulse. This scheme has been dubbed "Hybrid Chirped-Pulse Amplification", or HCPA[7].

To demonstrate a terawatt-class HCPA laser system, including compression to the Fourier-transform limit of the pulse, it is necessary to compare the spectral phase introduced by the OPCPA amplifier setup to that of the regen in the case of non-collinear, non-degenerate amplification. This is crucial for demonstrating the viability of OPCPA systems to produce ultrashort pulses: if the OPCPA stage were to introduce unusual spectral phase effect that could not be compensated for, short pulses would be unproducable. To study this, a comparison of pulses from a regen and an OPCPA amplifier, both going through the same multipass amplifier, was performed. The same oscillator, pulse stretcher, and regen discussed above were used. $35 \mathrm{~mm}$ of Ebarium-borate (BBO) material is needed to give enough gain length to get a $\sim 1 \mathrm{~nJ}$ seed pulse to an energy of $\sim 1 \mathrm{~mJ}$. In order to maximize the overlap between the pump and signal beams over the length of the material, two crystals are used: one is $10 \times 10 \times 20 \mathrm{~mm}$, the other is $10 \times 10 \times 15 \mathrm{~mm}$. This allows the two lengths to be aligned independently for maximum gain. The system is pumped with $280 \mathrm{~mJ}$ of $532 \mathrm{~nm}$ light, giving a pump intensity of $\sim 680 \mathrm{MW} / \mathrm{cm}^{2}$ in the non-linear crystals. Following the OPCPA stage, both the remaining pump light and the amplified signal beam are sent to a 4-pass Ti:Sapphire amplification stage. The pump energy arriving at the rod is $168 \mathrm{~mJ}$ after losses in both the OPCPA stage and transport. Following the 4-pass amplifier, the beam is sent to a pulse compressor for recompression.

Initially, the $1 \mathrm{~mJ}$ regen output was passed through the OPCPA crystals (with the pump mistimed to produce no gain) and amplified in the 4-pass amplifier to $55 \mathrm{~mJ}$ before being sent to the compressor. The measured pulse length is $61.2 \mathrm{fs}$ with a bandwidth of $15.76 \mathrm{~nm}$. This corresponds to a minimum pulse length of $60.4 \mathrm{fs}$, so the pulse is close to the transform limit. Subsequently, the regen light was attenuated to $2 \mathrm{~nJ}$ and amplified in the OPCPA stage to $0.5 \mathrm{~mJ}$. This beam was further amplified by the 4-pass amplifier to $25 \mathrm{~mJ}$ and compressed to $71 \mathrm{fs}$, with a retrieved bandwidth of $13.23 \mathrm{~nm}$. This can support pulses as short as $70 \mathrm{fs}$, so again this compressed pulse is near the transform limit. The spectral phases retrieved via GRENOUILLE measurements[8] are shown in Figure 3. The close similarity of the retrieved phases shows that no unusual (or uncompensatible) spectral phase is introduced by the OPCPA process, which demonstrates that the HCPA concept would be a viable replacement for the PLEIADES regenerative amplifier.

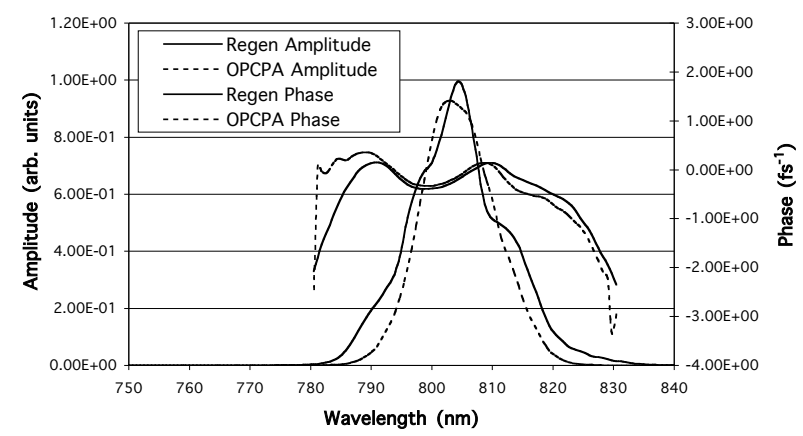

Figure 3: OPCPA and regenerative amplifier based systems spectral phase comparison

\section{REFERENCES}

1 D. J. Gibson, S. G. Anderson, C. P. J. Barty, et al., Phys. Plasmas 11, 2857 (2004).

2 R. Szipöcs, K. Ferencz, Ch. Spielmann, F. Krausz, Opt. Lett. 19, 201 (1994).

3 P. S. Banks, M. D. Perry, V. Yanovsky, et al., IEEE J. Quant. Elect. 36, 268 (2000).

4 J. Kuba, R. Shepherd, R. Booth, et al., Proc. SPIE, 5194, 183 (2004).

5 A. Piskarskas, D. Podenas, A. Stabinis, et al., in Ultrafast Phenomina V, G. R. Fleming, A. E. Siegman, eds., (Springer, Berlin, 1986) p. 142.

6 B. Wattellier, I. Jovanovic, C. P. J. Barty, in 2003 Conference on Lasers and Electro-Optics Technical Digest, paper CME1.

7 I. Jovanovic, C. A. Ebbers, C. P. J. Barty, Opt. Lett. 27 1622 (2002).

8 P. O'Shea, M. Kimmel, X. Gu, R. Trebino, Opt. Lett. 26, 932 (2001); P. O'Shea, M. Kimmel, R. Trebino, J. Opt. B 4, 44 (2002). 